(C) 1998 IEEE. Personal use of this material is permitted. Permission from IEEE must be obtained for all other uses, in any current or future media, including reprinting/republishing this material for advertising or promotional purposes, creating new collective works, for resale or redistribution to servers or lists, or reuse of any copyrighted component of this work in other works. 


\title{
Phase Noise and Sub-Carrier Spacing Effects on the Performance of an OFDM Communication System
}

\author{
Ana García Armada and Miguel Calvo, Member, IEEE
}

\begin{abstract}
This letter analyzes the phase noise effects on an orthogonal frequency division multiplexing (OFDM) signal and its dependence with the sub-carrier spacing. Pilot-based channel estimation, which has been suggested as a means of combating the channel effects, can also correct the phase noise effects under some circumstances, which are investigated.
\end{abstract}

\section{INTRODUCTION}

A MONG the activities of the latest European RACE and ACTS projects, there has been an increasing interest in Orthogonal Frequency Division Multiplexing (OFDM) as a means of combating impulsive noise and multipath effects, and making fuller use of the system available bandwidth. OFDM has been proposed for the Digital Television for Terrestrial Broadcasting System (dTTb) [1]. This system is now under test and two possible values are being considered for the number $N$ of sub-carriers: $2 \mathrm{~K}$ and $8 \mathrm{~K}$. Since the total bandwidth is fixed that means two different sub-carrier spacing values.

Both alternatives offer advantages and disadvantages: the greater the number of sub-carriers, the best protection against multipath delay spread, but on the other hand, phase noise effects must be considered.

Phase noise effects in OFDM have been studied by several authors. In [2] it is shown that OFDM is orders of magnitude more sensitive to carrier frequency offset and phase noise than single carrier systems. In [3] a synchronization scheme is studied using a digital phase-locked loop in the receiver to track a pilot signal. This corresponds to the technique implemented in DMT modems for ADSL [4].

As far as it concerns to dTTb [1], pilot-based channel estimation has been suggested as a means of combating the channel and phase noise effects. The purpose of this paper is to investigate the correction capabilities of this technique and to provide a means of selecting the sub-carrier spacing value given the phase noise characteristics of a system.

Since the constant phase error is assumed to be ideally corrected, the analysis of [2] is somehow optimistic. In order to provide more accurate results, an OFDM simulation system has been built using Alta Group's SPW. ${ }^{1}$

In the following the OFDM simulation system and the phase noise model will be described briefly After that, the nature of

The authors are with Grupo de Radiación, Dipartimento SSR, ETSI Telecomunicación, University Politecnica de Madrid, Ciudad Universitaria, 28040 Madrid, Spain (e-mail: ana@gr.ssr.upm.es).

\footnotetext{
${ }^{1}$ SPW (Signal Processing WorkSystem), Alta Group, Sunnyvale, CA Available HTTP: http://www.cadence.com.
}

phase noise effects on the OFDM signal reception will be analyzed.

Simulation results will be presented which allow one to investigate the phase noise effects and the system performance improvement achieved by pilot-based channel correction under different sub-carrier spacing and noise situations.

\section{OFDM SimULATION SYSTEM}

Orthogonal Frequency Division Multiplexing (OFDM) is one of the best alternatives to alleviate the multipath effects in mobile communications [5]. The spectrum associated to each elemental data in an OFDM system is a small portion of the total bandwidth, which is divided in $N$ sub-channels. Each of them is modulated with one symbol and they are all multiplexed in frequency.

The sampling frequency define in the dTTb [1] is $f_{s}=$ $64 / 7 \mathrm{MHz}=9.142857 \mathrm{MHz}$, which means that the values of the sub-carrier spacing $\Delta f=f_{s} / N$ and the OFDM symbol duration $T$ are

$$
\begin{array}{ll}
-8 \mathrm{~K}: & \Delta f=1.116 \mathrm{kHz}, T=896 \mu \mathrm{s} ; \\
-2 \mathrm{~K}: & \Delta f=4.464 \mathrm{kHz}, T=224 \mu \mathrm{s} ; \\
-128: & \Delta f=71.424 \mathrm{kHz}, T=14 \mu \mathrm{s} \text { (although } 128 \text { is } \\
& \text { not a number of sub-carriers used in the dTTb, it is } \\
\text { also considered in this paper to study its influenc } \\
\text { in the phase noise effects). }
\end{array}
$$

A complete OFDM simulation system block diagram has been built and pilot carriers have been inserted in the OFDM frame, as described in [6], for channel estimation and correction.

\section{Phase NoISE}

All superheterodyne receivers use one or more local oscillators to convert an input frequency to an intermediate frequency before the signal is demodulated. The local frequency before the signal is decreased except by improving OL's performance. Hence the importance of determining how much phase noise a receiver can admit maintaining the required performance.

Phase noise can be interpreted as a parasitic phase modulation in the oscillator's signal, which ideally would be a unique carrier with constant amplitude and frequency $f_{o}$. We have modeled it as a phase modulation of the carrier. The modulating signal is a white random process with a uniform or Gaussian pdf, which has been filtere so as to analyze the influenc of the phase noise correlation characteristics in the signal quality. 
Results are presented using the reciprocal of the filtere phase noise bandwidth: $T_{\phi}$ and comparing it to the OFDM symbol duration: $T_{\mathrm{OFDM}}$. The variance of the Gaussian phase noise after filterin (in radians ${ }^{2}$ ) is also used as a parameter.

\section{Theoretical Analysis of Phase Noise EFFECTS ON OFDM SIGNAL RECEPTION}

If $T$ represents the OFDM symbol duration, that is, $T=$ $N / B$, the complex envelope of the transmitted OFDM signal, for a given OFDM symbol, sampled with sampling frequency $f_{s}=1 / B$ is

$$
s(n)=\sum_{k=0}^{N-1} s_{k} \cdot e^{j \frac{2 \pi}{N} k n}, \quad \text { with } n=0,1, \cdots, N-1 .
$$

This symbol is actually extended with a cyclic prefi in order to cope with multipath delay spread. For the sake of simplicity, we will not consider this prefi since it is eliminated in the receiver.

Assuming that the channel is flat the signal is only affected by phase noise $\phi(n)$ at the receiver:

$$
r(n)=s(n) \cdot e^{j \phi(n)}
$$

and it is Orthogonal Frequency Division Demultiplexed by means of a discrete Fourier transform (DFT):

$$
\begin{aligned}
y(k) & =\frac{1}{N} \sum_{m=0}^{N-1} r(m) \cdot e^{-j \frac{2 \pi}{N} k m} \\
& =\frac{1}{N} \sum_{m=0}^{N-1} e^{j \phi(m)} \sum_{r=0}^{N-1} s_{r} \cdot e^{j \frac{2 \pi}{N} r m} e^{-j \frac{2 \pi}{N} k m} \\
& =\frac{1}{N} \sum_{m=0}^{N-1} e^{j \phi(m)} \sum_{r=0}^{N-1} s_{r} \cdot e^{j \frac{2 \pi}{N}(r-k) m} \\
& =\frac{1}{N} \sum_{r=0}^{N-1} s_{r} \sum_{m=0}^{N-1} e^{j \phi(m)} \cdot e^{j \frac{2 \pi}{N}(r-k) m}
\end{aligned}
$$

In order to separate the signal and noise terms, let us suppose that $\phi(m)$ is small, so that $e^{j \phi(m)} \approx 1+j \phi(m)$. In this case

$$
\begin{aligned}
y(k) & \approx \frac{1}{N} \sum_{r=0}^{N-1} s_{r} \sum_{m=0}^{N-1} e^{j \frac{2 \pi}{N}(r-k) m} \\
& +\frac{j}{N} \sum_{r=0}^{N-1} s_{r} \sum_{m=0}^{N-1} \phi(m) \cdot e^{j \frac{2 \pi}{N}(r-k) m} \\
& =s_{k}+\frac{j}{N} \sum_{r=0}^{N-1} s_{r} \sum_{m=0}^{N-1} \phi(m) \cdot e^{j \frac{2 \pi}{N}(r-k) m} \\
& =s_{k}+e_{k} .
\end{aligned}
$$

Thus we have an error term $e_{k}$ for each sub-carrier which results from some combination of all carriers and is added to the useful signal. Let us analyze more deeply this noise contribution:

a) If $r=k$ :

$$
\frac{j}{N} \sum_{r=0}^{N-1} s_{r} \sum_{m=0}^{N-1} \phi(m)=j \frac{s_{k}}{N} \sum_{m=0}^{N-1} \phi(m)=j \cdot s_{k} \cdot \Phi .
$$

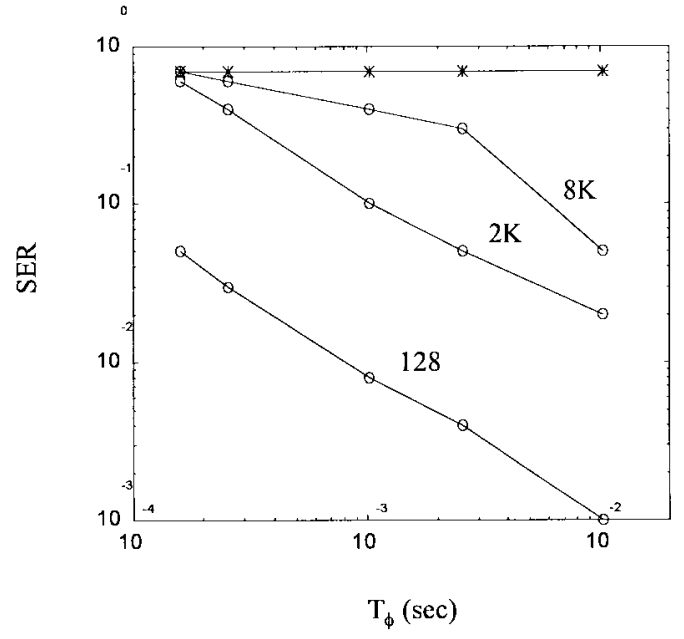

Fig. 1. OFDM with different numbers of sub-carriers- $*$ : corrupted, $\circ$ : corrected.

So we have a common error added to every sub-carrier which is proportional to its value multiplied by a complex number $j \Phi$, that is a rotation of the constellation.

This angle results from an average of phase noise over all of them (which implies low frequencies of phase noise spectrum) and, since it is constant for all sub-carriers, it can be corrected with the information provided by pilots [6].

b) If $r \neq k$ :

$$
\frac{j}{N} \sum_{\substack{r=0 \\ r \neq k}}^{N-1} s_{r} \sum_{m=0}^{N-1} \phi(m) \cdot e^{j \frac{2 \pi}{N}(r-k) m} .
$$

This term corresponds to the summation of the information of the $N-1$ sub-carriers each multiplied by some complex number which comes from an average of phase noise with a spectral shift. The result is also a complex number which is added to each sub-carrier's useful signal and has the appearance of white noise. It is normally known as intercarrier interference (ICI) or loss of orthogonality.

\section{ERROR PERFormances}

Simulations have been run using the system described in Section II so as to study the effects of phase noise in the reception of OFDM signals and the influenc of the sub-carrier spacing in the phase noise error correcting capabilities. The assumptions and simplification made in the previous analysis to understand the system behavior have not been applied to simulations, so results can be obtained for any phase noise and channel situations.

Fig. 1 shows the effects of uniform phase noise on the system performance. The symbol error rate (SER) degradation is the same for $128,2 \mathrm{~K}$, and $8 \mathrm{~K}$ systems. However, the correction capabilities depend on the phase noise to OFDM time ratios.

As it can be observed, phase noise effects can be corrected when the phase noise variations are much slower than the OFDM period. In this case the common phase error dominates over the ICI. 


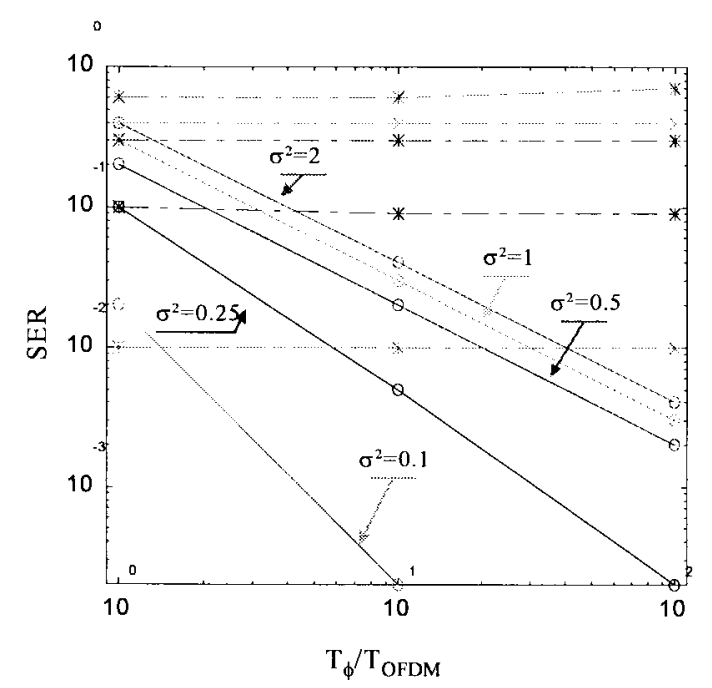

Fig. 2. $2 \mathrm{~K} \mathrm{OFDM}-*$ : corrupted signal, $\circ$ : corrected signal.

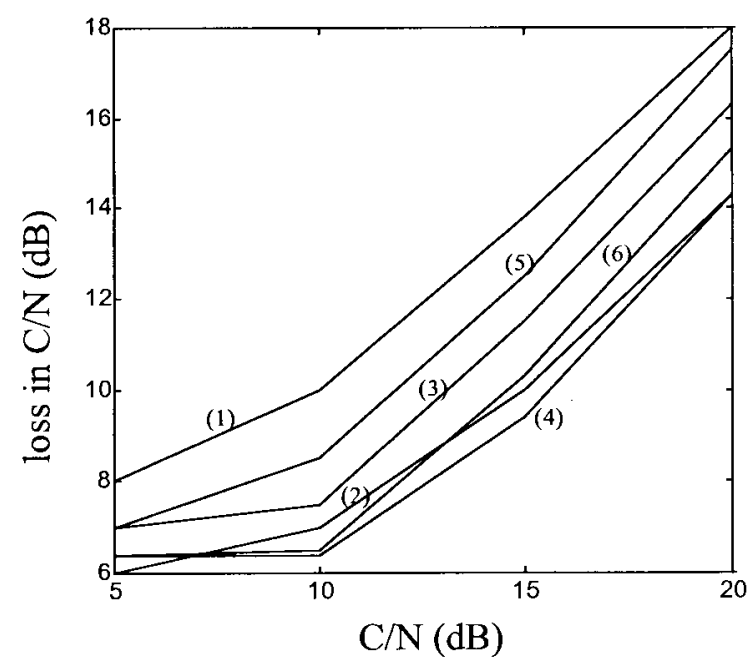

Fig. 3. $2 \mathrm{~K}$ OFDM-loss in $\mathrm{C} / \mathrm{N}$ after signal correction. (1) $\sigma^{2}=0.1, T_{\phi}=T_{\mathrm{OFDM}} ;$ (2) $\sigma^{2}=0.1, T_{\phi}=10 T_{\mathrm{OFDM}} ;$ (3) $\sigma^{2}=0.25, T_{\phi}=10 T_{\mathrm{OFDM}} ;$ (4) $\sigma^{2}=0.25, T_{\phi}=100 T_{\mathrm{OFDM}} ;$; $\sigma^{2}=0.5, T_{\phi}=10 T_{\mathrm{OFDM}} ;(6) \sigma^{2}=0.5, T_{\phi}=100 T_{\mathrm{OFDM}}$.

Fig. 2 presents the SER versus the ratio $T_{\phi} / T_{\mathrm{OFDM}}$ in the case of zero-mean Gaussian phase noise with its variance (in radians $^{2}$ ) as a parameter.

Again the system performance is strongly dependent on the ratio $T_{\phi} / T_{\mathrm{OFDM}}$. If the phase noise variations are faster than the OFDM signal, the correction scheme cannot cope with the phase noise errors and the corrected signal may exhibit an even worse SER than the noncorrected one (see $\sigma^{2}=0.1$ in Fig. 2).

Given that $T_{\phi} / T_{\mathrm{OFDM}}>1$, a better performance is achieved as the phase noise power decreases, with a greater improvement when noise variance is lower than 0.25 .

Once this correction is applied, the remaining degradation can be thought of as additional thermal noise power, which causes a loss in the carrier-to-noise ratio $(\mathrm{C} / \mathrm{N})$ when the modem is operating in an AWGN channel. This loss depends on the $\mathrm{C} / \mathrm{N}$ prior to the introduction of phase noise, as it is shown in Fig. 3.
While thermal noise is the dominant effect for low $\mathrm{C} / \mathrm{N}$ ratios, the system is more sensible to phase noise when the $\mathrm{C} / \mathrm{N}$ increases.

Depending on the phase noise characteristics of the oscillator and the desired SER performance, these curves allow the system designer to select the number of sub-carriers which can be used.

\section{CONCLUSIONS}

OFDM has been proposed and is being tested for the $\mathrm{dTTb}$ with the aim of combating multipath and making an efficien use of the available bandwidth. Pilot-based channel estimation has been suggested as a means of combating the channel effects. This technique can also correct the phase noise effects under some circumstances, as we have seen.

Since the number of sub-carriers to be used can affect the error performance in several ways, some alternatives have been investigated in relation to the phase noise effects. These have been identifie as two different error terms which affect the demultiplexed signal: a common phase rotation and the so called inter-symbol interference (ICI) or loss of orthogonality.

While a greater number of sub-carriers can offer a better protection against multipath delay spread, on the other hand, phase noise effects are worse in this situation. However we have shown for the proposed correction scheme that this is only true if the phase noise varies slowly with respect to the OFDM signal. Otherwise, the ICI dominates over the common phase error and the same performance is achieved in any case because the pilot-based channel correction will only compensate the latter.

SER curves have been provided which allow the system designer to choose an appropriated number of sub-carriers according to the desired symbol error rate and the system phase noise characteristics. It should be noted that the variance of the ICI term in Section IV could give an idea of the loss of performance caused by phase noise after applying the correction scheme, assuming that the common error is completely eliminated. This would be interesting in order to have some insight in the system performance without the need to run complete simulations. However, this topic is subject to further work.

\section{REFERENCES}

[1] dTTb Module 3, "System Specificatio for the Second dTTb Demonstrator," dTTb/M3/284, ver. 2.2, Feb. 1996.

[2] T. Pollet, M. van Bladel, and M. Moeneclaey, "BER sensitivity of OFDM systems to carrier frequency offset and Wiener phase noise," IEEE Trans. Commun., vol. 43, pp. 191-193, Feb./Mar./Apr. 1995.

[3] N. Zogakis and J. M. Cioffi "The effect of timing jitter on the performance of a discrete multitone system," IEEE Trans. Commun., vol. 44, pp. 798-808, July 1996.

[4] "Asymmetric Digital Subscriber Line (ADSL) Metallic Interface," ANSI Standard T1.413, 1995.

[5] L. J. Cimini, "Analysis and simulation of a digital mobile channel using orthogonal frequency division multiplexing," IEEE Trans. Commun., vol. COM-33, pp. 665-675, July 1985.

[6] A. García Armada, M. Calvo, and L. de Haro, "Influenc of the subcarrier spacing in the performance of an OFDM communication system," in Proc. HDTV'97, Montreux, Switzerland, 1997, pp. C1-C4. 